\title{
Development of an integrated CMOS DNA detection biochip
}

\author{
Yi-Ting Cheng, Chien-Ying Tsai, Ping-Hei Chen* \\ Department of Mechanical Engineering, National Taiwan University, Taipei 10617, Taiwan ROC \\ Received 8 November 2005; received in revised form 29 March 2006; accepted 30 March 2006 \\ Available online 4 May 2006
}

\begin{abstract}
This paper presents a CMOS DNA detection biochip using an electrical detection method with self-assembly multilayer gold nanoparticles (AuNPs). Each measuring spot of this biochip consists of three major parts; a pair of electrodes with a nanogap, a current amplifier circuit, and a heater with an embedded temperature sensor. The biochip is first fabricated by a TSMC (Taiwan Semiconductor Manufacturing Company Ltd.) $0.35 \mu \mathrm{m} 2 \mathrm{P} 4 \mathrm{M}$ standard CMOS process. Then, post-CMOS micromachining etch processes are used to expose the surface of the nanogap to test samples for the establishment of multilayer AuNPs through hybridization between single strand DNAs in the samples. The gap distance between a pair of electrodes is $350 \mathrm{~nm}$. Before taking DNA detection measurements, self-assembly monolayer AuNPs is established on the nanogap surface between two microelectrodes. Multilayer AuNPs can be observed if hybridization between single strand DNAs occurs. An approximately 1000-fold increase in electric current between the multilayer AuNPs over the monolayer AuNPs serves an indication of the presence of target DNA in test samples. After integrating the electrodes with an embedded current amplifier, the electric current of multilayer AuNPs is amplified to the order of $\mathrm{mA}$ that can be easily measured by a commercial Volt-Ohm-Milliammeter. The heating system with a heating element and a temperature sensor can be used to distinguish single base-pair mismatch hybridization from complementary hybridization for the establishment of multilayer AuNPs. The lowest detectable concentration of target DNA on this biochip is $0.1 \mathrm{nM}$.
\end{abstract}

(C) 2006 Elsevier B.V. All rights reserved.

Keywords: CMOS; DNA detection; Gold nanoparticles; Self-assembly; Current amplifier

\section{Introduction}

Determination of specific DNA sequences in biological samples can detect and identify certain infectious or inherited diseases or identify specific species. The purpose of DNA biochips development is to achieve rapid and reliable detection of specific DNA sequences from individual specific genes. Recently, with the rapid progress of modern technologies, especially nanotechnology, biotechnology and microelectronics, novel medical instruments have been proposed extensively. There is a trend to develop more immediate and inexpensive diagnostic devices. Thus, the development of an accurate, portable and relatively inexpensive biosensor has become one of the most important issues in the healthcare industry. The introduction of complementary metal oxide semiconductor (CMOS) chips for DNA

\footnotetext{
* Corresponding author at: Department of Mechanical Engineering, National Taiwan University, No. 1, Sec. 4, Roosevelt Rd., Taipei 10617, Taiwan ROC. Tel.: +8862 23670781; fax: +886223670781.

E-mail address: phchen@ntu.edu.tw (P.-H. Chen).
}

identification may overcome traditional problems and satisfy the requirements for inexpensive, accurate, and rapid detection because the fabrication processes for CMOS biochips are so mature that the detection circuit for CMOS biochips can be less than $1 \mu \mathrm{m}$. Many CMOS or CMOS-process compatible biochips have been introduced using two typical DNA detection methods, namely an optical approach [1-4] and an electrochemical approach [5-9].

Taton et al. [10-12] presented a DNA detection method by UV absorption of multilayered gold nanoparticles on a glass substrate. The detected signal can be amplified by the growth of gold nanoparticles through a chemical reduction of silver in the sample solution. The sensitivity of this detection method exceeds that of conventional fluorescence detection methods by two orders of magnitude in DNA identification. The same research group reported another novel DNA electrical detection method in 2002 [13]. They utilized the binding of oligonucleotide-functionalized gold nanoparticles in a $20 \mu \mathrm{m}$ gap between two electrodes to vary the impedance of the gap, and used a silver enhanced solution to amplify the originally undetectable signal. Tsai et al. [14] presented an electrical detection method that, instead 
of silver enhancement, made use of multilayer self-assembled gold nanoparticles for signal amplification between nanogap electrodes. The same group used a standard CMOS process to fabricate a DNA biochip for electrical detection of DNA in 2005
[15]. In the studies of Tsai's group [14,15], the detected electric current over multilayer gold nanoparticles through nanogap electrodes was at an order of $\mathrm{nA}$ when the concentration of the target DNA strand was low. Unfortunately, most detection meth-

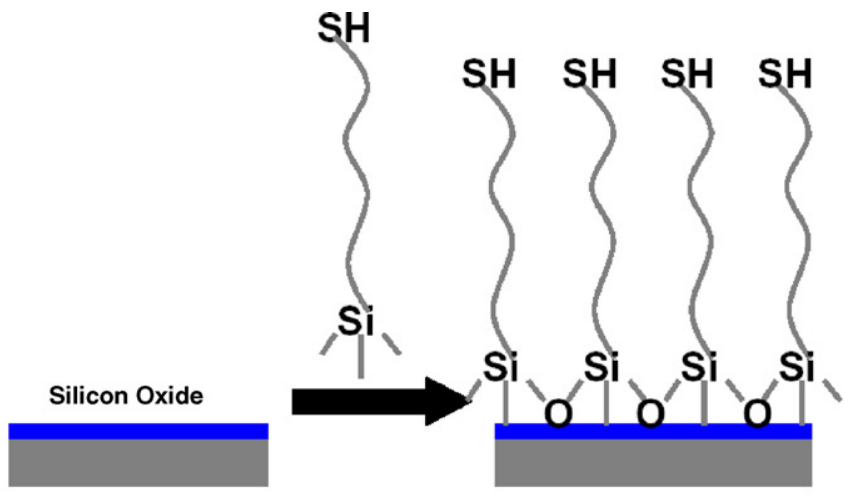

Si substrate
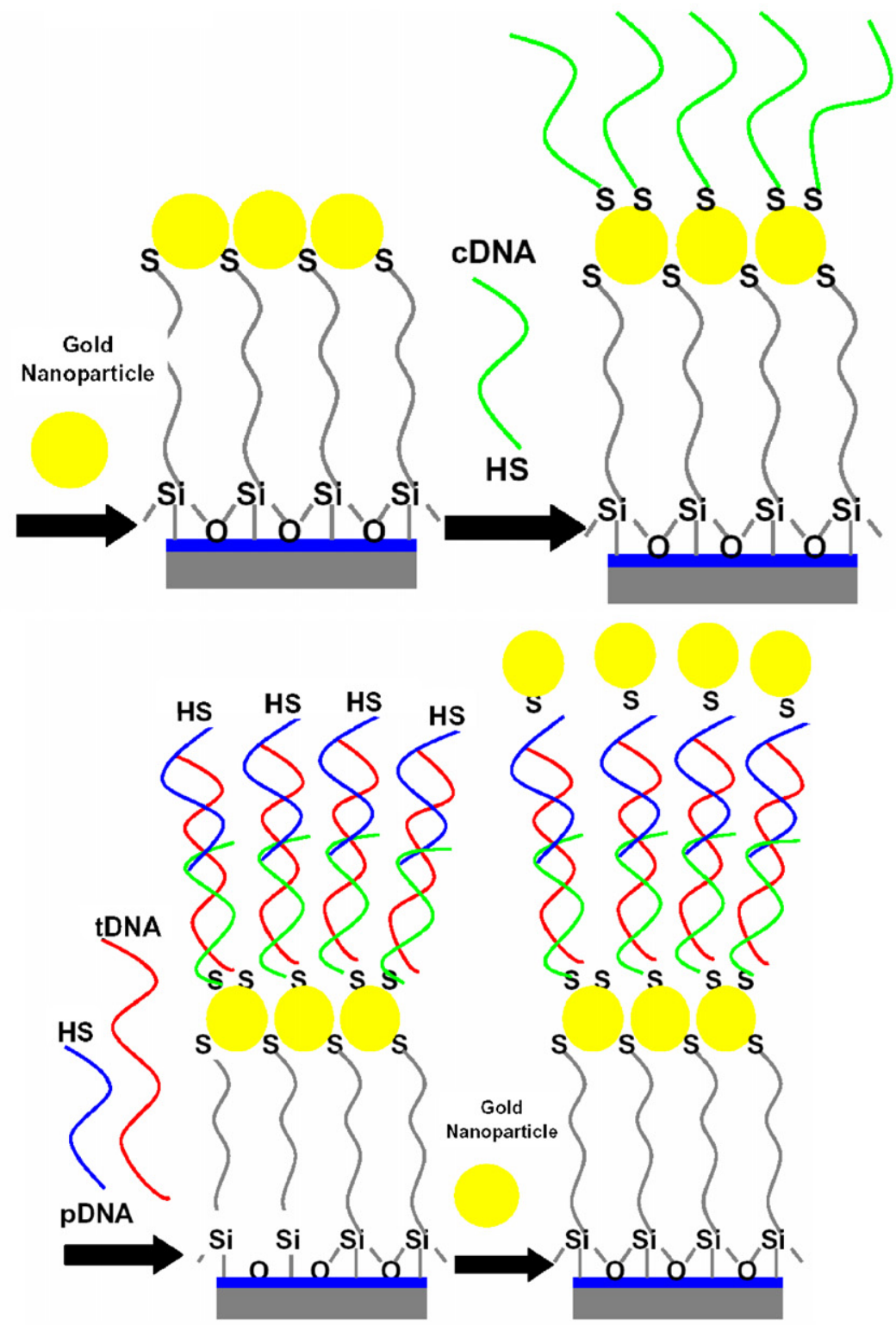

Fig. 1. Steps for establishing multilayer of self-assembly gold nanoparticles. 
ods mentioned above utilize expensive and complicated optical or electrical equipment, and are inconvenient for quick and inexpensive measurements.

In this study, cascode current mirrors are employed to amplify the low electric current, and a temperature system is used for single base-pair mismatch detection. The biochip is fabricated by a standard CMOS fabrication process combined with post-CMOS micromachining processes. The electrical detection method utilizing a self-assembly gold nanoparticle multilayer without silver enhancement is used to detect the hybridizations among probe, capture, and target oligonucleotides. Before taking DNA detection measurements, a self-assembly monolayer of AuNPs is established on a $\mathrm{SiO}_{2}$ surface between two microelectrodes. Then, capture oligonucleotide strands are immobilized on the top surface of the self-assembly monolayer of AuNPs. If target oligonucleotide strands are complementary with the probe and capture oligonucleotide strands in the solution, specific binding can occur among thiol-modified probe, capture, and target oligonucleotide strands. Finally, through a self-assembly process between suspended gold nanoparticles and the thiol-modified end of the probe oligonucleotide strand, multilayer AuNPs on the nanogap surface can be established. According to the method proposed by Tsai et al. [14], Fig. 1 illustrates the procedure to establish multilayer gold nanoparticles on the gap surface between two microelectrodes. With the integration of a current amplifier on the CMOS biochip, a commercial Volt-Ohm-Milliammeter can then be used for DNA identification.

\section{Experimental apparatus and experimental approach}

\subsection{Circuit design of current amplifier}

Fig. 2 shows the circuit design of five cascode current mirrors of the CMOS chip. One cascode current mirror is composed of four MOSFETs. The amplification factor of one cascode current mirror depends only on the ratio of $W / L$, where $W$ and $L$ denote the width and the length of the channel of MOSFET, respectively. After considering the range of input current signal, $i_{\mathrm{D} 1}$, and the range of load resistor, $R_{\text {load }}$, the amplification factor for each

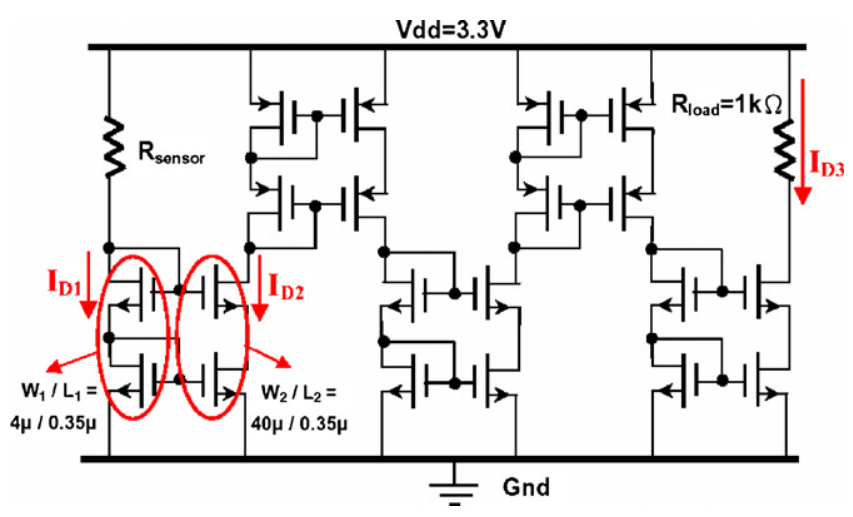

Fig. 2. Circuit design diagram of cascode current mirrors. cascode current mirror is chosen as:

$\frac{i_{\mathrm{D} 2}}{i_{\mathrm{D} 1}}=\frac{W_{2} / L_{2}}{W_{1} / L_{1}}=\frac{40 \mu \mathrm{m} / 0.35 \mu \mathrm{m}}{4 \mu \mathrm{m} / 0.35 \mu \mathrm{m}}=10^{1}$

where $i_{\mathrm{D}}$ is the current flowing through MOSFET from source to drain, the subscript 1 and 2 denote the input and amplified currents, respectively. After performing five stages of amplification, $i_{\mathrm{D} 3} / i_{\mathrm{D} 1}=10^{5}$ is obtained, where $i_{\mathrm{D} 1}$ is the input current signal from the detection electrodes and $i_{\mathrm{D} 3}$ is the output current signal for measurements.

\subsection{Detection principle of single base-pair mismatch hybridization}

When applied to base mismatch detection, hybridization assays are inherently limited in sensitivity. Detection of a point mutation in the test sequence (e.g., a small segment of genomic DNA) requires a distinguishable difference in pairing energies between a completely complementary and a mutated target strand. With only a single mutation in an extended oligonucleotide, the difference can be very small. In this detection system, temperature, or a thermal stringency wash, which is compatible with CMOS fabrication process, is the conventional tool for distinguishing single basepair (bp) mismatch.

Since the resistance of polysilicon increases with temperature, snack-like polysilicon layers in the standard CMOS process are used for heating and temperature sensing. Fig. 3 illustrates the design and micrograph of heating elements and temperature sensors. Poly 1 layer and poly 2 layer beneath the microelectrode represent heaters and temperature sensors, respectively. Poly 1 (heater) is an applied voltage to generate heat to denature target

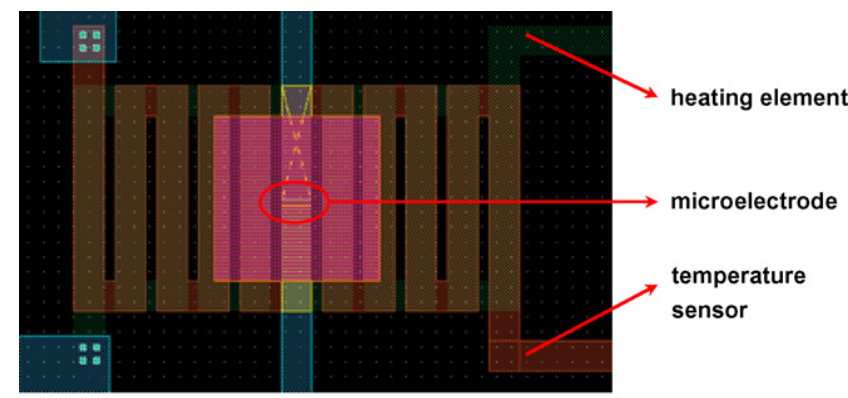

(a)

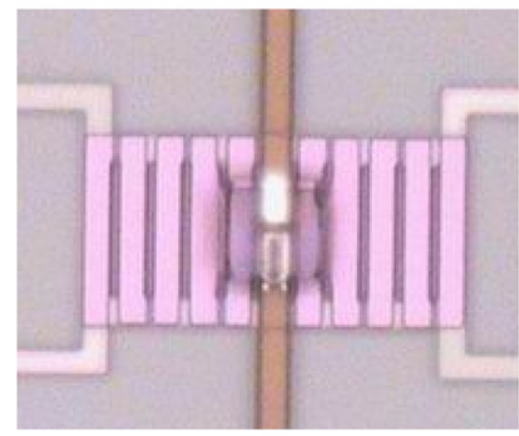

(b)

Fig. 3. (a) Design and (b) micrograph of heating elements and temperature sensors. Snack-like polysilicon layers are beneath the microelectrode. 
Table 1

Sequences of capture, probe, target and single basepair mismatch target oligonucleotide strands

\begin{tabular}{cc}
\hline \hline Name & Sequence \\
\hline cDNA & 3'-HS-AAAAAAAAAACCTAATAACAAT-5' \\
pDNA & 3'-TTATAACTATTCCTAAAAAAAAAAA-SH-5' \\
tDNA & 5'-GGATTATTGTTAAATATTGATAAGGAT-3' \\
single basepair & 5'-GGATTATdGTTAAATATTGATAAGGAT-3' \\
mismatch tDNA & \\
\hline \hline
\end{tabular}

DNA. After calibrating the relation between temperature and resistance of poly 2 (temperature sensor), we can monitor temperature change from the variation of resistance of poly 2 . Due to thermal dynamic properties, the melting temperature measured from temperature sensors of single bp mismatch target DNA will be less than that of perfect match target DNA. Sequences of capture, probe, target and single basepair mismatch target DNA are shown in Table 1.

\subsection{Post-CMOS processing of the CMOS chip}

The CMOS chip is composed of three major components; microelectrodes with a nanogap, current amplifier circuits, and a temperature system.

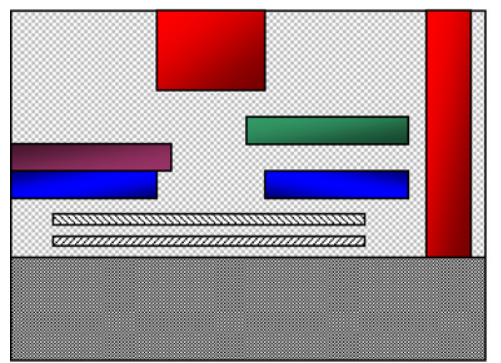

(a)

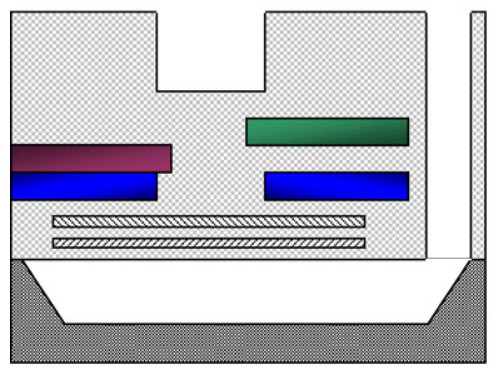

(c)

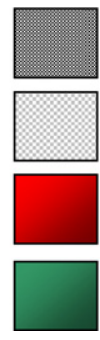

Si

$\mathrm{SiO}_{2}$

Al (etching hole)

Al
There are three etching steps to obtain microelectrodes with nanogap and thermal isolation for the temperature system on the chip simultaneously. The etching processes as a whole are realized by maskless etching. Fig. 4 shows the complete postCMOS micromachining process flow. The metal structures in the etching holes are removed by wet etching. For the current design of the CMOS biochip, the etching holes are used for etching the metal layer on top of the microelectrodes to decrease the time of dry etching.

The first step is to remove metal layers with traditional Piranha solution, a 3:1 mixture of concentrated sulfuric acid $\left(\mathrm{H}_{2} \mathrm{SO}_{4}\right)$ with hydrogen peroxide $\left(\mathrm{H}_{2} \mathrm{O}_{2}\right)$, at $80{ }^{\circ} \mathrm{C}$ for $30 \mathrm{~min}$. The isotropic wet etching solution flows and etches along the etching holes that are originally filled with metal structures.

Then, a 35:1 mixture of concentrated tetra-methyl ammonium hydroxide (TMAH) with an ammonium carbonate $\left(\left(\mathrm{NH}_{4}\right)_{2} \mathrm{CO}_{3}\right)$ solution is used to remove the silicon substrate for thermal isolation. The CMOS chip is immersed in the TMAH solution at $100{ }^{\circ} \mathrm{C}$ for $15 \mathrm{~min}$.

The final step is to employ anisotropic dry etching-reaction ion etching (RIE, model: RIE-10N, SAMCO) to remove $\mathrm{SiO}_{2}$ with a recipe by using gas flows of $\mathrm{CF}_{4}$ at a flow rate of $20 \mathrm{sccm}$ and $\mathrm{O}_{2}$ at a flow rate of $5.2 \mathrm{sccm}$ under a vacuum pressure of $7 \mathrm{~Pa}$ with an RF power of $100 \mathrm{~W}$ for $60 \mathrm{~min}$.

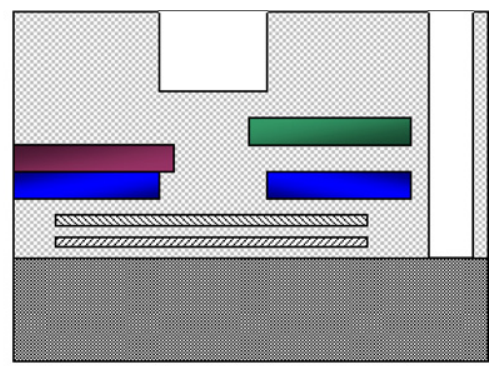

(b)

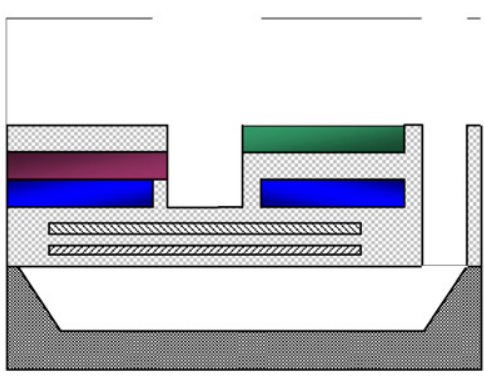

(d)

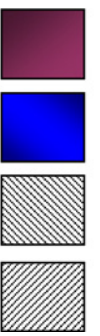

Tungsten (via)

Al (microelectrode)

Poly 2 (temp. sensor)

Poly 1 (heater)

Fig. 4. Post-CMOS micromachining process flow: (a) cross-sectional view of CMOS biochip; (b) wet etching for removing etching hole; (c) TMAH etching for removing Si substrate; (d) RIE dry etching for reducing the thickness of $\mathrm{SiO}_{2}$ to form the microelectrodes. 


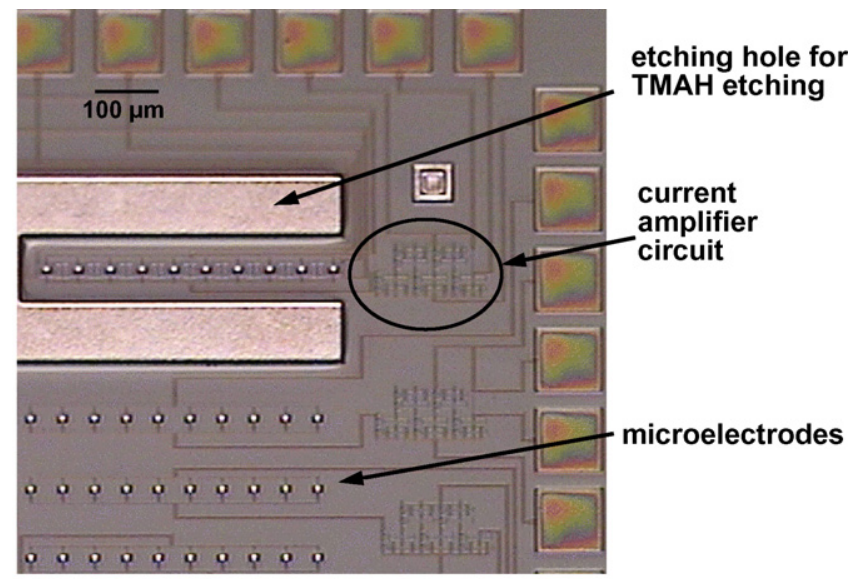

(a)

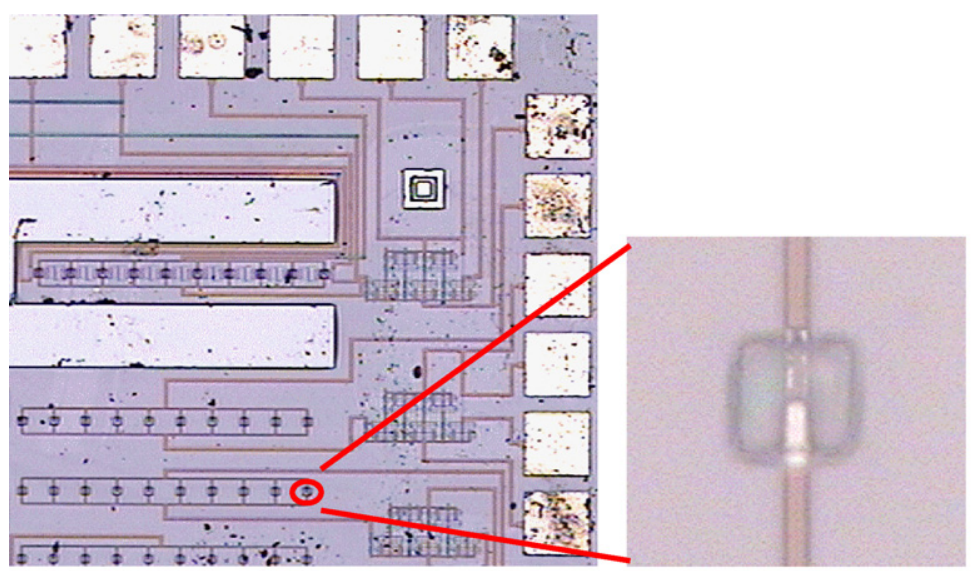

(b)

Fig. 5. Micrograph of the CMOS biochip (a) before and (b) after post-CMOS etching processes and the enlargement of a microelectrode.

Since dry etching gas exhibits high selectivity between aluminum and $\mathrm{SiO}_{2}$, the metal layers can be used as an etching mask. Due to anisotropic etching of $\mathrm{SiO}_{2}$ by RIE, the pattern of microelectrodes is formed defined by the layers of metal 2 and via 1 . Microelectrodes lie on the layer of metal 1 . The reaction ion etching continues until the microelectrodes appear. The surface of the nanogap between microelectrodes is still made of $\mathrm{SiO}_{2}$ for DNA immobilization. Fig. 5(a) and (b) illustrate the micrograph of the CMOS biochip before and after postmicromachining process, respectively. This chip is fabricated by TSMC $0.35 \mu \mathrm{m}$ 2P4M standard CMOS process, with a dimension of $1 \mathrm{~mm} \times 3 \mathrm{~mm}$, and there are 80 measuring spots on the biochip. The nanogap is $350 \mathrm{~nm}$ in width, $5 \mu \mathrm{m}$ in length, and $2 \mu \mathrm{m}$ in thickness.

\section{Results}

Fig. 6(a) and (b) show the FE-SEM micrographs of gold nanoparticle monolayer and multilayer immobilized in the gap between two microelectrodes, respectively. The white spots in the micrographs are gold nanoparticles. The distribution density of gold nanoparticles of the multilayer is much larger than that of the monolayer. It can be observed that the gold nanoparticles are discretely scattered and not continuously distributed on the gap. Fig. 7(a) and (b) show the $I-V$ characteristic curves of the monolayer and multilayer gold nanoparticle structure over a $350 \mathrm{~nm}$ gap without current amplifier circuit, respectively. In this study, we use the semiconductor device analysis system (HP 4156) to measure the electrical signals. The noise level of this machine is about several fA and the scanning rate for the $I-V$ curve measurement is $10 \mathrm{mV} / \mathrm{s}$. After successful immobilization of the gold nanoparticle monolayer on the gap, the electric current passing through the two microelectrodes is lower than 10 pA (Fig. 7(a)) with an applied voltage of $1 \mathrm{~V}$. Although gold nanoparticles do not contact directly with each other, the current passing through the microelectrodes is still detectable. It is assumed to be the result of the tunneling effect through isolated gold nanoparticles. The measured $I-V$ curve for the gold nanoparticle monolayer follows the Ohm's law with some fluctuations. After hybridization among oligonucleotide strands over the gold nanoparticle monolayer, the electric current passing through two microelectrodes for the multilayer of self-assembly gold nanoparticle increases to a value of $10 \mathrm{nA}$ at an applied voltage of $1 \mathrm{~V}$ (Fig. 7(b)). It is noted that the concentration of target 


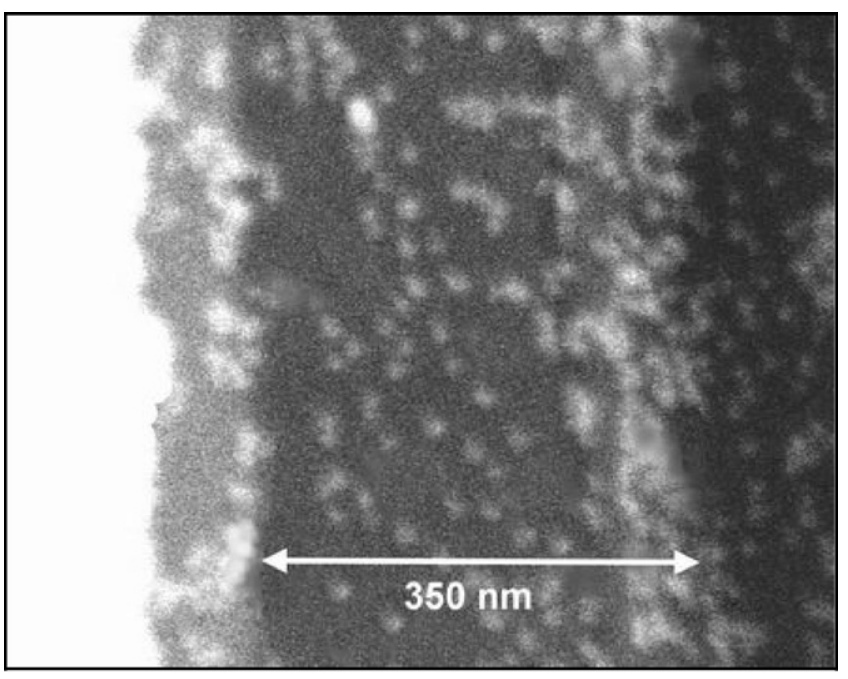

(a)

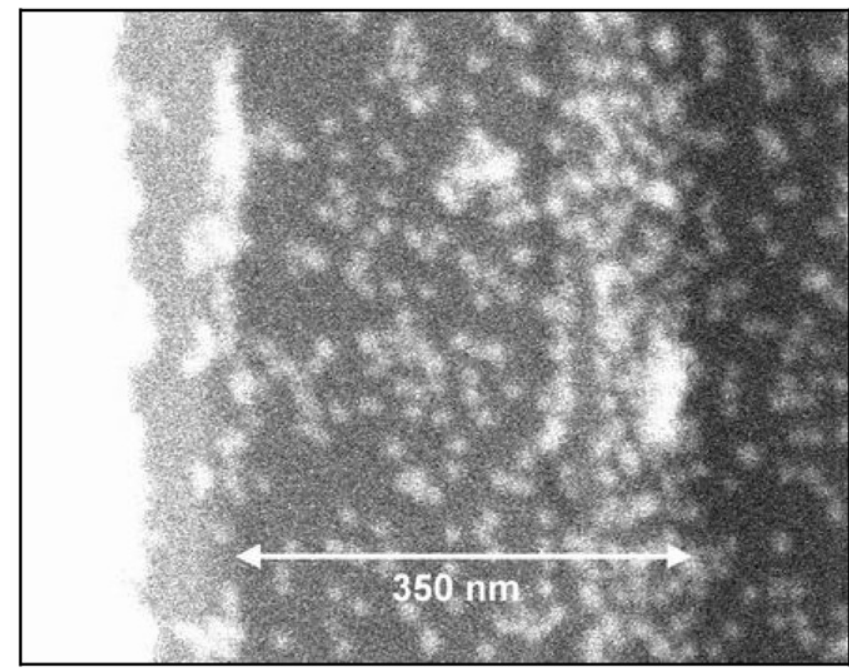

(b)

Fig. 6. FE-SEM micrograph of self-assembly layered gold nanoparticles over the nanogap between microelectrodes: (a) monolayer and (b) multilayer.

oligonucleotides in the sample solution is $10 \mathrm{nM}$. Fig. 8 shows the current signals of different target DNA concentrations. Since the current signal of the target DNA concentration of $0.01 \mathrm{nM}$ is as low as that of the monolayer gold nanoparticle structure, it proves that the lowest detectable target DNA concentration is $0.1 \mathrm{nM}$.

In the temperature system, the calibration curve of the relation between temperature and resistance of poly 2 (temperature sensor) is shown in Fig. 9. The resistance of poly-silicon increases quite linearly with temperature. Fig. 10 illustrates current signals versus melting temperatures of single bp mismatch and that of perfect match of target DNA that can be distinguished by the $2{ }^{\circ} \mathrm{C}$ difference in melting temperature. The current signal of perfect match target DNA decreases dramatically at $48^{\circ} \mathrm{C}$, but that of single bp mismatch target DNA occurs at $46^{\circ} \mathrm{C}$. From this result, we can find out single mutation of target DNA precisely.

After combining the microelectrodes with the current amplifier circuit, the current signals of the monolayer and multilayer
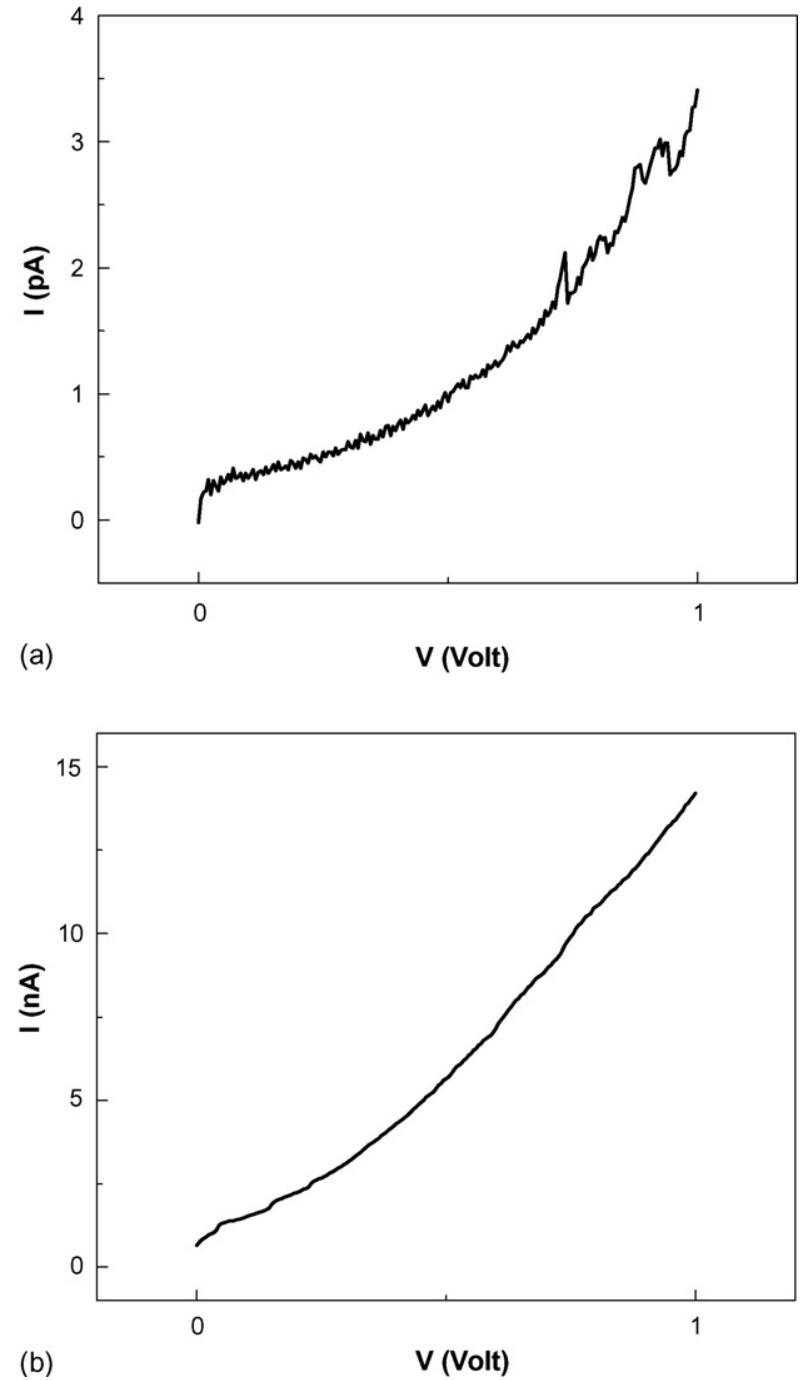

Fig. 7. $I-V$ characteristic curves of self-assembly layered AuNPs over a $350 \mathrm{~nm}$ nanogap without current amplification at a target DNA concentration of $10 \mathrm{nM}$ : (a) monolayer and (b) multilayer. The scanning rate is $10 \mathrm{mV} / \mathrm{s}$.

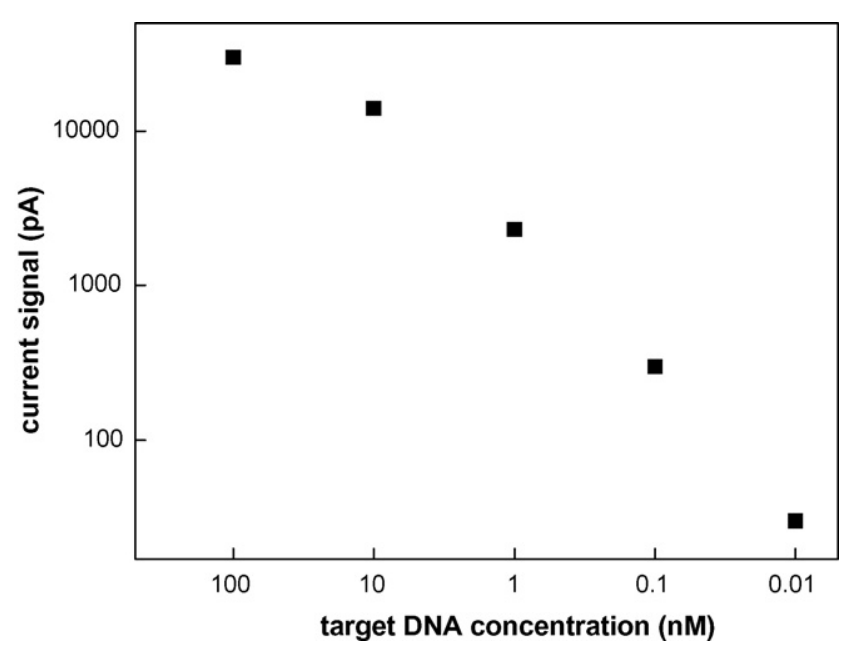

Fig. 8. Electric currents of multilayer AuNPs without current amplification at different target DNA concentrations with an applied voltage of $1.0 \mathrm{~V}$. 


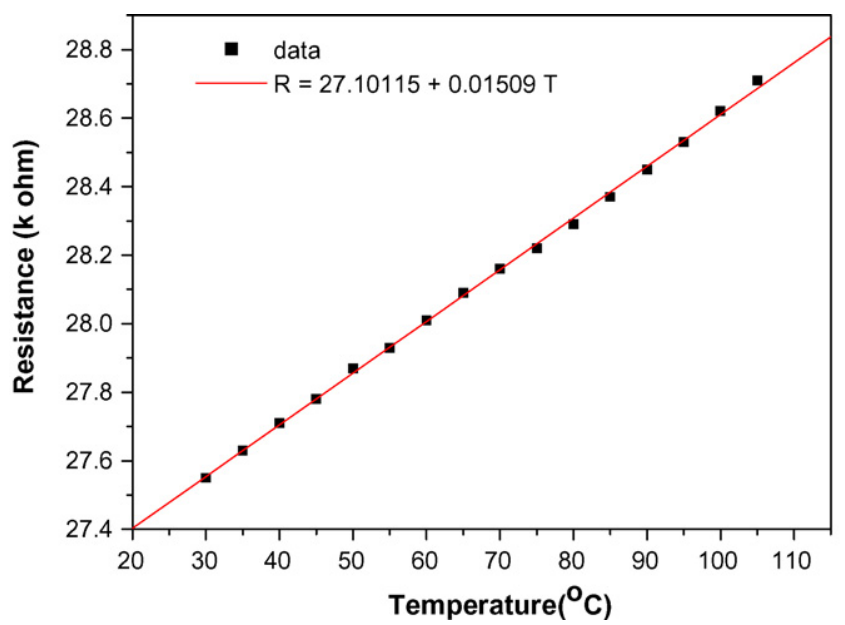

Fig. 9. Calibration curve of temperature and electrical resistance of poly 2 that serves as a temperature sensor.

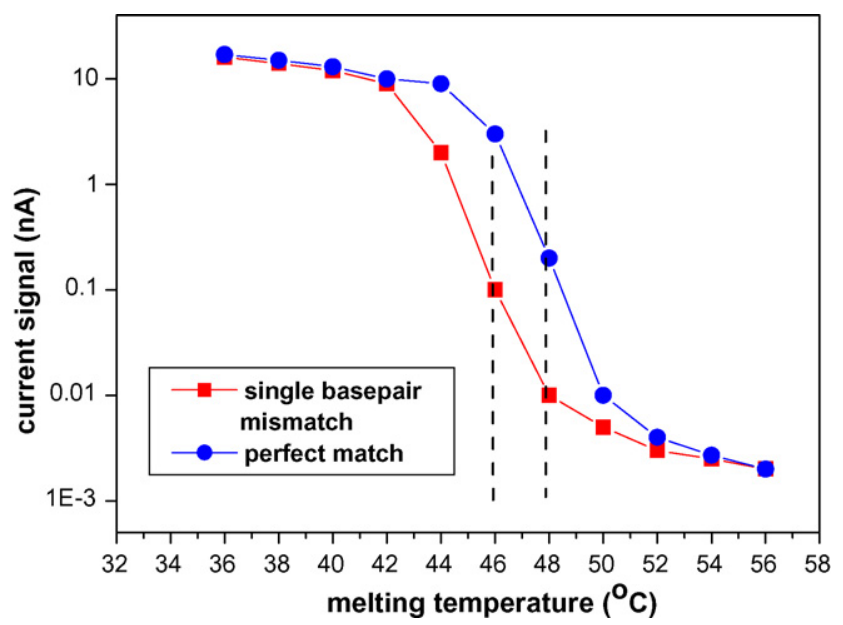

Fig. 10. Current signals vs. melting temperature for both single bp mismatch and complementary hybridization between single strand DNAs. The concentration of target DNA in the test sample is $10 \mathrm{nM}$.

gold nanoparticle structure are successfully amplified to the level of $1 \mu \mathrm{A}$ and $1 \mathrm{~mA}$, respectively. Fig. 11 illustrates the comparison of the current signals of the monolayer and multilayer gold nanoparticle structure over a $350 \mathrm{~nm}$ nanogap with and with-

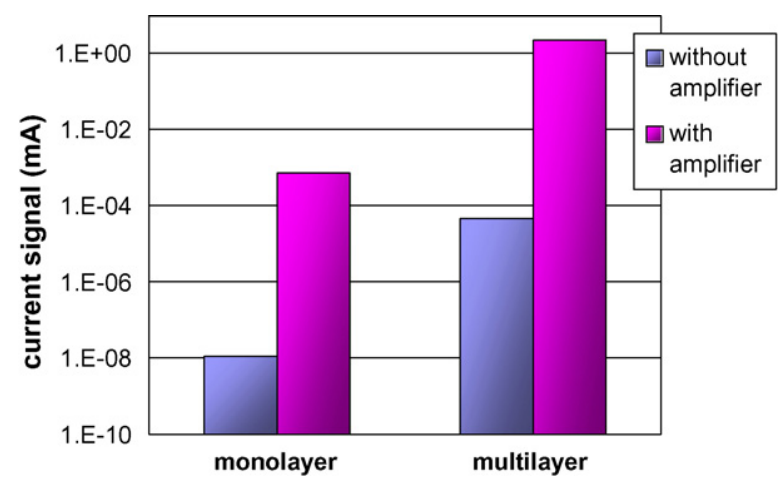

Fig. 11. Current signals of monolayer and multilayer self-assembly gold nanoparticles over a $350 \mathrm{~nm}$ nanogap with and without a current amplifier circuit at a target DNA concentration of $10 \mathrm{nM}$. out the current amplifier circuit. The electric signal $(\mathrm{mA})$ of the multilayer gold nanoparticle structure over the nanogap could be detected by a commercial Volt-ohm-Milliammeter. The current mirrors play a key role in the CMOS biochip. The noise produced in regular electronic devices would render it impossible to detect the current signal at a pico-ampere level unless the signals can be amplified without noise interference. Usually, pico-ampere level electric signal can only be detected with some extremely sensitive instrument (e.g. HP 4156 Semiconductor Device Analysis System). In this study, the current mirrors have been effective in amplifying the signal through both the monolayer and multilayer gold nanoparticles, and thus have allowed us to obtain the current through multilayer gold nanoparticles with a common commercial Volt-ohm-Milliammeter.

\section{Conclusion}

In this study, a DNA detection system including DNA biosensors, a current amplifier circuit, and a temperature system for single basepair mismatch detection is designed and developed for DNA identification by electrical detection. A novel technique using a self-assembly method combined with cascode current mirrors to amplify electrical signals is presented. The electric current passing through the gold nanoparticle multilayer, which is formed by complementary target oligonucleotide strands, exceeds that through the gold nanoparticle monolayer by three orders of magnitude. After being amplified by the current mirrors, the electrical signals of the multilayer gold nanoparticle structure over a nanogap can be measured by a commercial Volt-ohm-Milliammeter. With temperature sensors and heaters, single mutation of target DNA could be distinguished precisely. We have demonstrated that the combination of CMOS biosensor structures, IC design technique and commercially available electronic devices is useful to identify specific DNA sequences. CMOS process, which is widely applied to digital and ana$\log \mathrm{IC}$, is very common and standard in the semiconductor industry. This means that CMOS based instruments by mass production can be of relatively low cost for the healthcare industry. The characteristics of CMOS chips make this device very attractive to be developed as personalized clinical diagnostic instruments.

\section{Acknowledgements}

The authors deeply appreciate CIC (Chip Implementation Center) for providing all the design tools, TSMC for fabrication, and equipment for post-micromachining by NSC MEMS Research Center, Taiwan. We are grateful for the financial support provided by National Science Council in Taiwan under the grant number of NSC 94-2212-E-002-049. The work in this study could not have been achieved without their support.

\section{References}

[1] P. Swanson, R. Gelbart, E. Atlas, L. Yang, T. Grogan, W.F. Butler, D.E. Ackley, E. Sheldon, A fully multiplexed CMOS biochip for DNA analysis, Sens. Actuators B: Chem. 64 (2000) 22-30. 
[2] J.M. Yang, J. Bell, Y. Huang, M. Tirado, D. Thomas, A.H. Forster, R.W. Haigis, P.D. Swanson, R.B. Wallace, B. Martinsons, M. Krihak, An integrated, stacked microlaboratory for biological agent detection with DNA and immunoassays, Biosens. Bioelectron. 17 (2002) 605-618.

[3] P.E. Lobert, D. Bourgeois, R. Pampin, A. Akheyar, L.M. Hagelsieb, D. Flandre, J. Remacle, Immobilization of DNA on CMOS compatible materials, Sens. Actuators B: Chem. 92 (2003) 90-97.

[4] J.H. Chen, T.F. Chen, R.S. Huang, J. Gong, J.C. Li, W.C. Chen, T.H. Hseu, I.C. Hsu, A novel micro-well array chip for liquid phase biomaterial processing and detection, Sens. Actuators A: Phys. 108 (2003) 193-200.

[5] J. Albers, T. Grunwald, E. Nebling, G. Piechotta, R. Hintsche, Electrical biochip technology—a tool for microarrays and continuous monitoring, Anal. Bioanal. Chem. 377 (2003) 521-527.

[6] J. Li, M. Xue, Z. Lu, Z.K. Zhang, C.G. Feng, M.S. Chan, A high-density conduction-based micro-DNA identification array fabricated with a CMOS compatible process, IEEE Trans. Electr. Dev. 50 (2003) 2165-2170.

[7] L. Moreno-Hagelsieb, P.E. Lobert, R. Pampin, D. Bourgeois, J. Remacle, D. Flandre, Sensitive DNA electrical detection based on interdigitated $\mathrm{Al} / \mathrm{Al}_{2} \mathrm{O}_{3}$ microelectrodes, Sens. Actuators B: Chem. 98 (2004) 269274.

[8] C. Guiducci, C. Stagni, G. Zuccheri, A. Bogliolo, L. Benini, B. Samori, B. Riccò, DNA detection by integrable electronics, Biosens. Bioelectron. 19 (2004) 781-787.

[9] D.S. Kim, Y.T. Jeong, H.J. Park, J.K. Shin, P. Choi, J.H. Lee, G. Lim, An FET-type charge sensor for highly sensitive detection of DNA sequence, Biosens. Bioelectron. 20 (2004) 69-74.

[10] T.A. Taton, C.A. Mirkin, R.L. Letsinger, Scanometric DNA array detection with nanoparticle probes, Science 289 (2000) 1757-1760.

[11] T.A. Taton, R.C. Mucic, C.A. Mirkin, R.L. Letsinger, The DNA-mediated formation of supramolecular nano- and multilayered nanoparticle structures, J. Am. Chem. Soc. 122 (2000) 6305-6306.

[12] T.A. Taton, G. Lu, C.A. Mirkin, Two-color labeling of oligonucleotide arrays via size-selective scattering of nanoparticle probes, J. Am. Chem. Soc. 123 (2001) 5164-5165.
[13] S.J. Park, T.A. Taton, C.A. Mirkin, Array-based electrical detection of DNA with nanoparticle probes, Science 295 (2002) 15031506.

[14] C.Y. Tsai, Y.H. Tsai, C.C. Pun, B. Chan, T.Y. Luh, C.C. Chen, F.H. Ko, P.J. Chen, P.H. Chen, Electrical detection of DNA hybridization with multilayer gold nanoparticles between nanogap electrodes, Microsyst. Technol. 11 (2005) 91-96.

[15] Y.T. Cheng, C.C. Pun, C.Y. Tsai, P.H. Chen, An array-based CMOS biochip for electrical detection of DNA with multilayer self-assembly gold nanoparticles, Sens. Actuators B: Chem. 109 (2005) 249255 .

\section{Biographies}

Yi-Ting Cheng received his BSc degree in mechanical engineering from National Taiwan University in 2001 and is currently pursuing the $\mathrm{PhD}$ degree from Nation Taiwan University in Taipei, Taiwan ROC. His main research interests include MEMS fabrication, CMOS-MEMS design, biochip for DNA detection, and microfluid system.

Chien-Ying Tsai received his $\mathrm{PhD}$ degree from National Taiwan University in 2004. In 2005, he worked as a postdoctoral research fellow at the Department of Mechanical Engineering in National Taiwan University. His research interests include MEMS with emphasis on the mechanical properties of thin films using micromachined structures, CMOS process and self-assembly gold nanoparticle structure.

Ping-Hei Chen received his BSc degree from National Taiwan University in 1980, and PhD degree from University of Minnesota in 1988. After graduation, he joined the Department of Mechanical Engineering of National Taiwan University as an Associate Professor. He was promoted to a Full Professor in 1996. His research areas include micro-thermal-fluid systems, Lab-on-a-chip for DNA detection, Nanofluid, MEMS fabrication technology, and cooling devices for electronic equipment. 